\title{
Progressive transition from pre-planned to intraoperative optimizing seed implant: post implementation analysis
}

\author{
Hsiang-Chi Kuo, PhD, Ass. Prof., William Bodner, MD, Ass. Prof., Ravindra Yaparpalvi, MSc, Chandan Guha, MD, PhD, Prof., \\ Bhupendra M. Tolia, MD, Keyur J. Mehta, MD, Ass. Prof., Dennis Mah, PhD, Shalom Kalnicki, MD, Prof. \\ Montefiore Medical Center, Albert Einstein College of Medicine, Department of Radiation Oncology, Montefiore Medical Center. \\ New York, USA
}

\begin{abstract}
Purpose: To perform a dosimetric comparison between a pre-planned technique and a pre-plan based intraoperative technique in prostate cancer patients treated with I-125 permanent seed implantation.

Material and methods: Thirty patients were treated with I-125 permanent seed implantation using TRUS guidance. The first 15 of these patients (Arm A) were treated with a pre-planned technique using ultrasound images acquired prior to seed implantation. To evaluate the reproducibility of the prostate volume, ultrasound images were also acquired during the procedure in the operating room (OR). A surface registration was applied to determine the $6 \mathrm{D}$ offset between different image sets in arm A. The remaining 15 patients (Arm B) were planned by putting the pre-plan on the intraoperative ultrasound image and then re-optimizing the seed locations with minimal changes to the pre-plan needle locations. Post implant dosimetric analyses included comparisons of $\mathrm{V}_{100}$ (prostate), $\mathrm{D}_{90}$ (prostate) and $\mathrm{V}_{100}($ rectum).

Results: In Arm A, the 6D offsets between the two image sets were $\theta_{\mathrm{x}}=-1.4 \pm 4.3 ; \theta_{\mathrm{y}}=-1.7 \pm 2.6 ; \theta_{\mathrm{z}}=-0.5 \pm 2.6$; $X=0.5 \pm 1.8 \mathrm{~mm} ; \mathrm{Y}=-1.3 \pm-3.5 \mathrm{~mm} ; \mathrm{Z}=-1.6 \pm 2.2 \mathrm{~mm}$. These differences alone degraded $\mathrm{V}_{100}$ by $6.4 \%$ and $\mathrm{D}_{90}$ by $9.3 \%$ in the pre-plan, respectively. Comparing Arm A with Arm B, the pre-plan based intraoperative optimization of seed locations used in the plans for patients in Arm B improved the $\mathrm{V}_{100}$ and $\mathrm{D}_{90}$ in their post-implant studies by $4.0 \%$ and $5.7 \%$, respectively. This was achieved without significantly increasing the rectal dose $\left(\mathrm{V}_{100}\right.$ (rectum)).

Conclusions: We have progressively moved prostate seed implantation from a pre-planned technique to a pre-plan based intraoperative technique. In addition to reserving the advantage of cost-effective seed ordering and efficient OR implantation, our intraoperative technique demonstrates increased accuracy and precision compared to the pre-planned
\end{abstract} technique.

Key words: pre-plan, intraoperative planning, seed implant, prostate cancer.

\section{Purpose}

Accurate and reproducible prostate ultrasound volume acquisition, precise implantation of the needle and placing seeds guided by an optimized plan are essential requirements to perform high quality prostate implants. Pre-planning ultrasound studies have value in that anatomic variation such as pubic arch interference and the presence or absence of median lobe hypertrophy can be evaluated in the treatment position [1]. The three-dimensional size and volume of the prostate can be accurately measured, verifying coverage of the ultrasound template and allowing a costefficient ordering of seeds. However, performing pre-plans for prostate implants has the disadvantage that planning volumes in the pre-plan may differ from those obtained in the operating room (OR). In addition to the difficulty of reproducing the setup position, a part of the difference may result from OR anesthesia causing relaxation of pelvic musculature and consequently changing the shape of the prostate. Additionally, the prostate may undergo volume changes in the interval between pre-planning and implantation for other reasons.

In 2009, our institute was seeking a progressive transition from a pre-planned technique $\left(\mathrm{P}_{\mathrm{I}}\right)$ to an intraoperative technique. As a result, a pre-plan based intraoperative technique was developed which consists of applying the preplan to an intraoperative ultrasound image followed by an optimization of seed locations with minimal changes to preplan needle locations. This new method $\left(\mathrm{P}_{\mathrm{II}}\right)$ preserves the aforementioned cost and time efficiency of the pre-plan technique while increasing the quality of the implantation. The improvement of the new technique over the previous method is quantified by comparing dose metrics from post 
implementation CT analysis. We also compared our results with other major intraoperative techniques [2-9] - intraoperative preplanning $\left(\mathrm{O}_{\mathrm{I}}\right)$, interactive planning $\left(\mathrm{O}_{\mathrm{II}}\right)$, and dynamic dose calculation $\left.\left(\mathrm{O}_{\mathrm{III}}\right)\right)$ in terms of post implantation dosimetric results and efficiency.

Changes in the shape of the prostate and the resulting loss of geometric fidelity in the implantation procedure can degrade the quality of the implant. This study also investigated the effect these changes have on both implantation techniques. By applying a surface registration to preoperative and intraoperative ultrasound prostate volumes, the reproducibility of the ultrasound prostate volumes and its impact on the dose distribution was analyzed. We quantified the degradation in geometric fidelity between the volumes and reported the ability of our new pre-plan based intraoperative technique to mitigate these effects.

\section{Material and methods}

Between $10 / 2009$ to $04 / 2011$, thirty consecutive prostate cancer patients were treated in our institution using Transrectal Ultrasound-guided (TRUS) I-125 permanent seed implantation. Thirteen $(43 \%)$ of the patients had received $45 \mathrm{~Gy}$ to the whole pelvis prior to the implantation via external beam radiation therapy. Six $(20 \%)$ of the patients had received hormonal therapy prior to radiation therapy. These thirty patients were divided into two arms to study the dosimetric impact between the pre-planned with and without re-optimization in the OR. Each patient was placed in the extended dorsal lithotomic position and a bi-planar ultrasound probe (B\&K Medical, model 8558, Wilmington, MA, USA) was inserted into the rectum. Ultrasound images were acquired at $5 \mathrm{~mm}$ steps and transferred directly to the VariSeed ${ }^{\mathrm{TM}}$ planning system for treatment planning (Version 7.1, Varian Medical System, Palo Alto, CA, USA).

\section{Pre-planned technique}

The first 15 patients (Arm A) were treated with a preplanned technique which has been explained in detail elsewhere [10-12]. In short, it requires a detailed ultrasound mapping of the prostate some weeks (typically two weeks) before the implant itself. Instead of using uniform loading, we planned using the modified peripheral loading technique. On the day of treatment, after anesthesia and a setup check via ultrasound image, the brachytherapist executes the needle insertion and seed implantation by adhering to the preplan done before surgery. No further ultrasound image acquisition was needed for the pre-planned technique. While each patient in Arm A was treated utilizing this technique, for study purpose an additional ultrasound image set was acquired.

\section{Intra-operative optimizing pre-planned technique}

The next 15 patients (Arm B) were treated by re-optimizing the pre-plan in the OR. After the acquisition of the ultrasound image and target delineation, the brachytherapist inserted the needles based on the pre-plan needle locations and the physicist covered the pre-planned seed distribution simultaneously. A re-optimization of the seed distribution follows to account for any changes in prostate position and volume of images acquired in the OR.

\section{Post implant dosimetric study}

For both arms, post implant CT scans were obtained 30 days after the implant. To minimize the effects of the inter-observer variation, all of the prostate contours in CT were delineated by the same physician (WB). By comparing the CT image ( $2.5 \mathrm{~mm}$ slice thickness) with the TRUS image (5 mm slice thickness), puboretalis muscles and anterior venous plexus were excluded from prostate contour. Rectum includes the entire rectal wall and inner mucosa was delineated $1 \mathrm{~cm}$ above and below base and apex of prostate, respectively. Cumulative dose-volume histograms (DVHs) were generated for each plan. Plan evaluation was based on RTOG guidelines and its outcome from multi-institute analyses $[13,14]$. Post plan dosimetric results were evaluated by the percentage volume of the prostate receiving $100 \%$ and $150 \%$ of the prescription dose $\left(\mathrm{V}_{100}, \mathrm{~V}_{150}\right)$ and the minimum percentage of the prescribed dose received by $90 \%$ of the prostate gland $\left(\mathrm{D}_{90}\right)$. Rectal dose was evaluated in terms of the volume of the rectum receiving $100 \%$ of the prescribed dose $\left[\mathrm{V}_{100}\right.$ (rectum)]. Currently our implantation is planned with modified peripheral technique with needle loading at least $1 \mathrm{~cm}$ away from the boarder of urethra plus the planned $D_{90}$ is less than $103 \%$ of the prescribed dose, urethra area was less than $110 \%$ of the prescribed dose. As this dose level at urethra is much less than the dose constraint of $150 \%$ prescribed dose, urethral dose results are not discussed in this study. Results of our current study are compared with the major intra-operative planning techniques (Table 1, Fig. 1) [15] in terms of dosimetric coverage and efficiency.

\section{Statistical comparisons of the two techniques}

Two hypotheses were made in the present study. First, the two techniques were the same in terms of pre-planning results. To allow possible outliers and non-normal distributions of testing parameters in both arms, Mann-Whitney tests (non-parametric equivalent to independent $t$-test) were applied to compute the Z-scores (sample size > 10) of each testing parameter. By using a two-tailed test, the hypothesis is not true if $|z|>1.96$ such that the observed $z$ is significant at the $5 \%$ level. The second hypothesis is that the modified technique has better post dosimetric coverage. The same z-score is computed and tested against a one-tailed distribution. It is true if $|z|>1.64$ such that the observed $\mathrm{z}$ is significant at the $5 \%$ level.

\section{Reproducibility between volume studies}

The pre-plan volume study was utilized as the reference and a pre-plan was done with this image set. A second ultrasound volume study was acquired in the operating room prior to the seed insertion. The prostate volume was contoured for each set of ultrasound images and was exported from the VariSeed (Varian) TPS. For each set of the prostate volume images, the posterior portion of the prostate base closest to the center of the ultrasound probe was taken as the reference point. A $3 \mathrm{D}$ point set surface registration was 
Table 1. Terminology of the techniques compared in this report

\begin{tabular}{ll} 
Planning modality & \multicolumn{1}{c}{ Description } \\
\hline $\begin{array}{l}\text { Pre-planning } \\
\text { (PI) }\end{array}$ & Creation of a plan a few days or weeks before the implant procedure. Plan executed without modification \\
\hline $\begin{array}{l}\text { Intraoperative optimized } \\
\text { pre-planning }\end{array}$ & Re-optimize preplan by adapting the volume change in OR while needles are inserting \\
(PII) & Creation of a plan in the OR just before the implant procedure, with immediate execution of the plan \\
\hline $\begin{array}{l}\text { Intraoperative planning } \\
\text { (OI) }\end{array}$ & $\begin{array}{l}\text { Stepwise refinement of the treatment plan using computerized dose calculations derived from image-based } \\
\text { needle position feedback }\end{array}$ \\
\hline $\begin{array}{l}\text { Interactive planning } \\
\text { (II) }\end{array}$ & Constant updating of calculations of dose distribution using continuous deposited seed position feedback \\
\hline $\begin{array}{l}\text { Dynamic dose calculation } \\
\text { (OIII) }\end{array}$
\end{tabular}

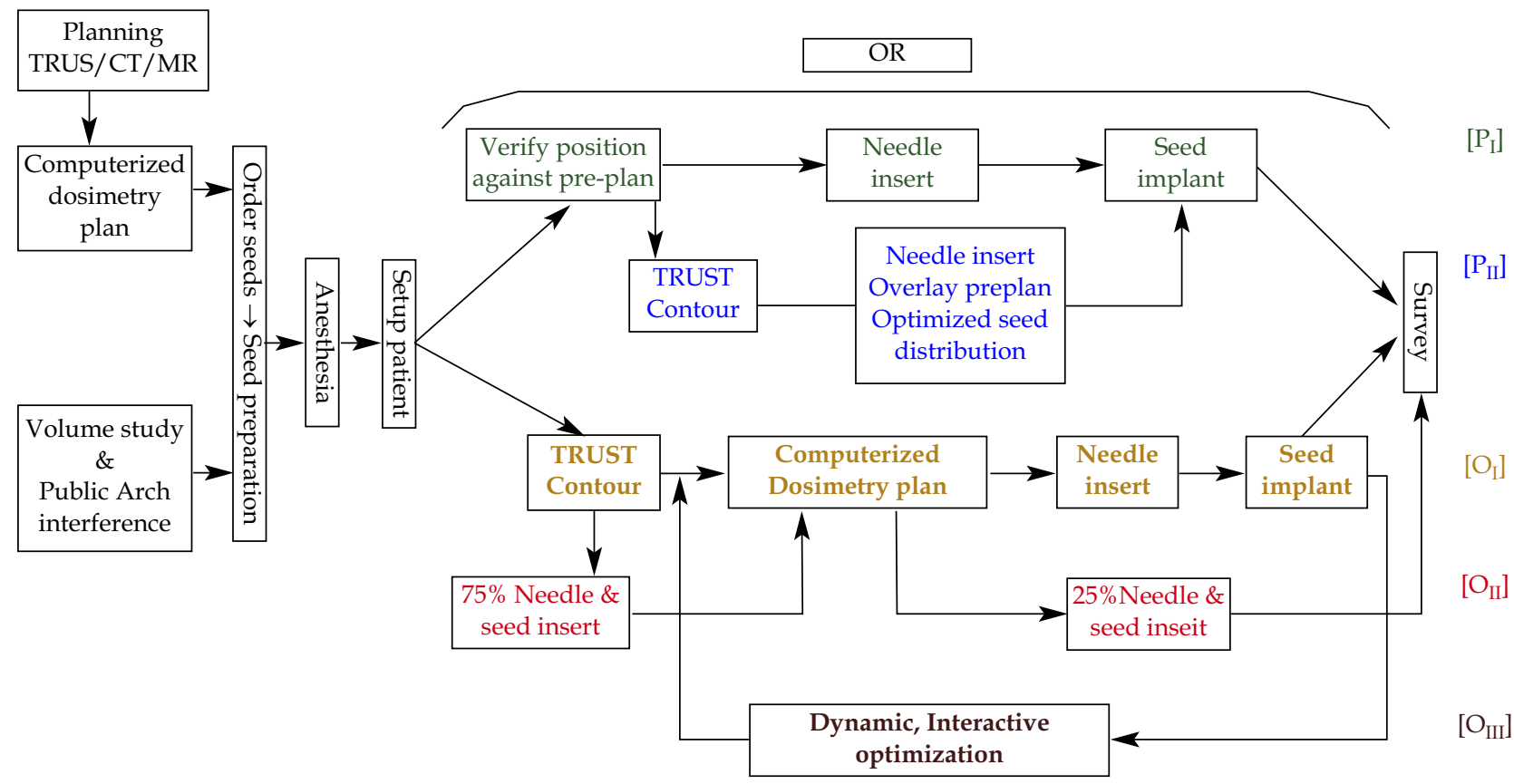

Fig. 1. Flow chart of different pre-planed $(\mathrm{P})$ and intraoperative $(\mathrm{O})$ prostate implantations

performed between the pre-plan prostate volume and the intra-op volume. An in-house software utilized an interactive closest points (ICP) algorithm to find the correspondence of the points between two volumes. Specifically, a $6 \mathrm{D}$ (three dimensional space translations plus three angle rotation with respect to the three axes) Euclidian transformation was optimized to find the best fit between the two volumes. The reproducibility of the prostate volume in $6 \mathrm{D}$ and its impact on the dose coverage for the intra-op volumes were analyzed. To study the dosimetric effect of the uncertainty caused by the $6 \mathrm{D}$ offset between different volume images, the pre-planned seed distribution was overlaid on the acquired images in the OR and new planned dose was calculated.

A limitation of the ICP algorithm here is that the 6D Euclidian transformation is based on the assumption of rigid body and does not consider the shape change between volumes acquired before and in OR.

\section{Results}

Figure 2 shows a box-plot of the 6D offset between the two TRUS image sets acquired before the OR for the fifteen patients we compared in Arm A. The 6D offsets between the two images (expressed by mean \pm standard deviation) were $\theta_{\mathrm{x}}=-1.4 \pm 4.3^{\circ} ; \theta_{\mathrm{y}}=-1.7 \pm 2.6^{\circ} ; \theta_{\mathrm{z}}=-0.5 \pm 2.6^{\circ}$; $X=0.5 \pm 1.8 \mathrm{~mm} ; Y=-1.3 \pm-3.5 \mathrm{~mm} ; Z=-1.6 \pm 2.2 \mathrm{~mm}$. Table 2 summarizes the dosimetric results from the different implantations. The mean prostate volumes measured from TRUS and CT are similar in both arms. The planning $\mathrm{V}_{100}$ and $\mathrm{D}_{90}$ in both Arms meet the criteria of $90 \%$ and $100 \%$, respectively. In Arm A, after overlaying the seed distribution from the pre-plan onto the second set of ultrasound images acquired in the OR, the $\mathrm{V}_{100}$ and $\mathrm{D}_{90}$ of the preplan were degraded by $6.4 \%$ and $9.3 \%$, respectively (Arm A* in Table 2). These degradations make some of patients in Arm A have $\mathrm{D}_{90}$ in post implant plan less than $90 \%$. The 
z-scores of $\mathrm{D}_{90}$ and $\mathrm{V}_{100}$ in the pre-implantation set of Table 2 are -0.35 and -0.26 indicating both arms are the same in terms of dosimetric coverage. The z-score of $\mathrm{D}_{90}$ and $\mathrm{V}_{100}$ of post plan set of Table 2 are -2.09 and -1.70 showing a significant difference at the $5 \%$ level. The range and distribution of dosimetric results in each group is displayed in Fig. 3. Smaller ranges (SD $\sim 2 \%$ ) were seen in the pre-implantation set (either pre-plan or OR plan) for each arm. In the post plan set, Arm B shows smaller range ( $4.5 \%$ vs. $8 \%$ in Arm A) and has less deviation from the pre-plan. Table 3 demonstrates the dosimetric results from the different techniques. To be concise, the table only lists the results from the pioneers of each technique $[2,7,8]$ and compares the results of our current study. Each technique has no variation from the prescription dose (as defined from RTOG's $\mathrm{D}_{90}>90 \%$ criteria $[13,14]$ ) except the results from our preplan approach which has a mean value with minor variation $\left(90 \%>\mathrm{D}_{90}>80 \%\right)$. The total loading seed strength is low in our current implantation $(0.97 \mathrm{U} / \mathrm{cc})$ compared to $1.10 \mathrm{U} / \mathrm{cc}, 1.08 \mathrm{U} / \mathrm{cc}$ and $1.32 \mathrm{U} / \mathrm{cc}$ for techniques utilized by Seattle, Matzkin, and Mt. Sinai, respectively. This indicates a slight under loading of total seed strength in our implantation compared to other published techniques.

\section{Discussion}

This study demonstrates a progression from a preplanned implant technique to intraoperative optimization.
The study design includes a two phase investigation for a transition toward an intraoperative prostate implantation technique. In the initial phase, no attempt was made to change the seed distribution generated from the volume study before OR. Rather, we were interested in determining whether the prostate volume acquired in the OR was similar to the prostate volume acquired in the pre-plan volume study. The second phase is a controlled use of a different seed loading implementation. Both Arms have been compared and have similar pre-plan dosimetric coverage in terms of $\mathrm{D}_{90}$ and $\mathrm{V}_{100}$ and are statistically indistinguishable.

The 15 ultrasound image sets acquired in the OR from Arm A showed a significantly different orientation and position from the image sets acquired during the volume study for the pre-plan. The intraoperative prostate volume differed slightly $(\sim 10 \%)$ compared to the pre-plan prostate volume. Patients who had their initial OR appointment postponed showed larger volume change due to a longer time lapse between the volume study and the OR. Some patients showed pubic arc interference in the OR and needed angling the ultra-sound probe. This reduced the pressure to the gland causing a shape change in volume. The precision limits of contouring on ultrasound may contribute to the volume difference between the two volumes acquired before and in the OR. Combined with the 6D offset (standard deviation of $4.3^{\circ}, 2.6^{\circ}, 2.6^{\circ}, 1.8 \mathrm{~mm}, 3.5 \mathrm{~mm}$ and $2.2 \mathrm{~mm}$ in $\theta_{x}, \theta_{y}, \theta_{z}, X, Y$, and $Z$ direction, respectively) between the two volumes, $\mathrm{V}_{100}$ and $\mathrm{D}_{90}$ of the prostate volume in the
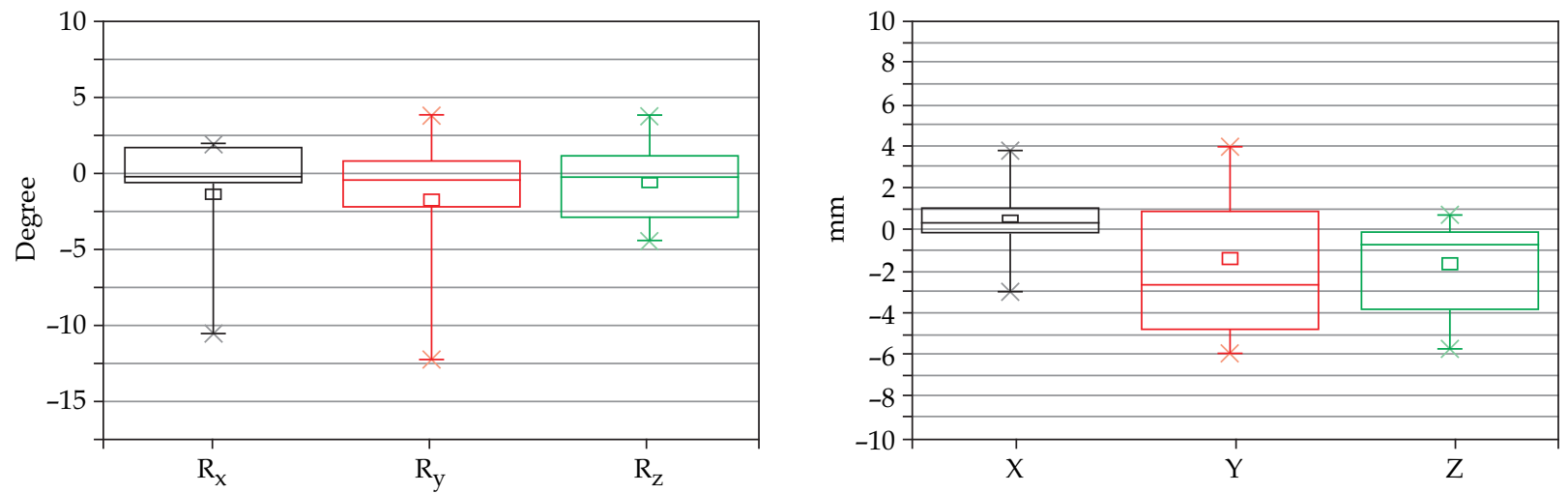

Fig. 2. Box-plot of the $6 \mathrm{D}$ offset between the two TRUS image sets acquired before OR and during implantation for the patients we compared in Arm A. $R_{x}, R_{y}$ and $R_{z}$ are the $\theta_{x}, \theta_{y}$, and $\theta_{z}$ in text, respectively. The bottom and top of the box is the $25^{\text {th }}$ and $75^{\text {th }}$ percentile, respectively. The band across box is the median and the square is the mean. The error bars represent the range

Table 2. Summary of the analysis in this study. Z-scores are computed between Arm A and B

\begin{tabular}{lcccc} 
& Arm A & Arm A* & Arm B & Z-score \\
\hline Cases & 15 & 15 & 15 & \\
\hline TRUS (cc) & $34.1 \pm 15.3$ & $37.5 \pm 16.3$ & $34.9 \pm 11.7$ & \\
\hline CT (cc) & $32.8 \pm 13.1$ & $32.8 \pm 13.1$ & $33.8 \pm 9.6$ & -0.26 \\
\hline $\mathrm{V}_{100}$ (planning, \%) & $90.3 \pm 1.5$ & $83.9 \pm 1.5$ & $90.4 \pm 0.7$ & -0.35 \\
\hline $\mathrm{D}_{90}$ (planning, \%) & $100.4 \pm 2.1$ & $91.1 \pm 6.8$ & $100.7 \pm 1.2$ & -1.7 \\
\hline $\mathrm{V}_{100}$ (post, \%) & $81.8 \pm 7.4$ & $81.8 \pm 7.4$ & $85.8 \pm 3.7$ & -2.09 \\
\hline $\mathrm{D}_{90}$ (post, \%) & $87.7 \pm 8.9$ & $87.7 \pm 8.9$ & $93.4 \pm 5.2$ &
\end{tabular}


Table 3. Comparison of post implant CT-based dosimetric parameters with different implantation

\begin{tabular}{|c|c|c|c|c|c|c|}
\hline Technique & $P_{1}$ & $P_{1}$ & $\mathrm{P}_{\|}$ & $\mathrm{O}_{1}$ & $\mathrm{O}_{\|}$ & $\mathrm{O}_{\text {III }}$ \\
\hline Study & Seattle & Current & Current & Matzkin & Mt. Saini & MSKCC \\
\hline$U / C c^{*}$ & 1.10 & 0.97 & 0.97 & 1.08 & 1.32 & \\
\hline $\mathrm{V}_{100}(\%)$ & 94 & 81.8 & 85.8 & 95.2 & 94 & 94 \\
\hline $\mathrm{V}_{150}(\%)$ & 47 & 44.4 & 50.4 & 45.4 & 56 & 32 \\
\hline $\mathrm{D}_{90}(\%)$ & 107 & 87.7 & 93.4 & 115 & 121 & 105 \\
\hline OR min. & $\sim 60$ & $60 \sim 90$ & $60 \sim 100$ & $>90$ & $>120$ & $>120$ \\
\hline
\end{tabular}

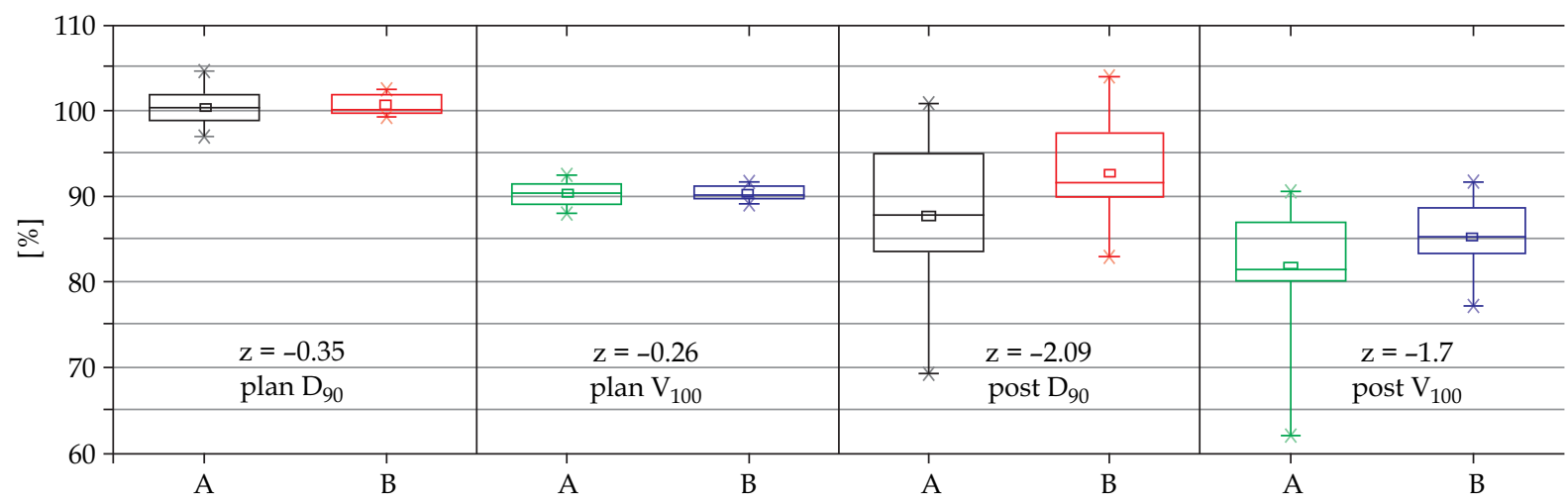

Fig. 3. Box-plot of $D_{90}$ and $V_{100}$ with pre-plan or OR plan vs. post plan for Arm $A$ and Arm B, respectively. $|z|>1.96$ and $|z|>1.64$ indicate a significant difference at the 5\% level for a two-tailed test and a one-tailed test, respectively

OR decreased by $6.4 \%$ and $9.3 \%$, respectively. This implies that the dosimetric coverage of the pre-plan on the volume study image set is different from the dosimetric coverage of the same pre-plan on the OR volume image set (z-score -4.5 and -4.13 for $D_{90}$ and $V_{100}$, respectively) due to the reproducibility of prostate volume image in the OR.

The second phase of the study was to adapt the volume change in the OR and modify the seed distribution simultaneously in the planning computer while the needle pattern of the pre-plan is being used for insertion. Although the cases in our study have a $10 \%$ volume increase in the OR compared to the volume study, we found that with the same total number of seeds as the pre-plan, the re-optimization of the seed distribution enabled us to reshape the dose distribution upon viewing the OR image and make the target coverage similar to the pre-plan. Part of the reason is that the target of our pre-plan is a PTV with a $3 \mathrm{~mm}$ margin to the prostate. Occasionally, we needed to alter the needle placement to recover the cold island due to a major volume change; most of the time, re-optimizing the seed distribution (up to 10 20\% of the original seed locations) will make the coverage meet the same criteria of $\mathrm{D}_{90} \sim 100 \%$ and $\mathrm{V}_{100} \sim 90 \%$ (no significant difference with z-score of -0.39 and -0.33 , respectively).

Compared to the original pre-planned technique, this new implantation technique only takes minimal additional time to acquire OR ultrasound image and delineate target, plus a little time for re-optimization if it cannot be completed within the time of needle insertion. The overall planning time increased approximately 10 minutes. The time increase can be reduced by another 2-3 minutes if planning system can support loading the pre-plan automatically to the OR image set. Re-optimization of pre-plan is normally done by the time when brachytherapist completes needle insertions based on the pre-planned pattern. In contrast, it will be at least 20-30 minutes more (compare to pre-plan technique) for an intraoperative pre-planned technique [3] to start a computerized plan in the OR before the needle and seed implantation. Another 30 minutes on top of this will be added if it is to perform real time interactive adjustments of the needle and seed position $[4,8]$.

The post CT dosimetric results of this new implantation technique showed the overall degradation of $\mathrm{V}_{100}$ and $\mathrm{D}_{90}$ from the OR plan was $4.6 \%$ and $7.3 \%$, respectively. This degradation is close to Stone's report [5] of $4.8 \%$ and 3\%, respectively or Ishiyama's recent report [16] of $1.1 \%$ and $7.9 \%$, respectively. This indicates that our seed implementation with a modified pre-plan has compatible results compared to other implementation utilizing the real time interactive optimization technique. In terms of the improvement with the modified pre-plan to the pre-planned technique, the 15 consecutive controlled pre-planned patients in $\operatorname{Arm} \mathrm{A}, \mathrm{V}_{100}$ and $\mathrm{D}_{90}$ were $81.8 \%$ and $87.7 \%$, respectively; the following 15 consecutive OR optimization implantations 
demonstrated the dosimetric results of $\mathrm{V}_{100}$ and $\mathrm{D}_{90}$ to $85.8 \%$ and $93.4 \%$, respectively. Z-scores of -1.70 and -2.09 on $V_{100}$ and $\mathrm{D}_{90}$ show that our OR re-optimization is a statistically significant improvement over pre-plan technique. There are other studies that show a different degree of improvement by comparing pre-planned and intraoperative optimization techniques. Matzkin et al. [2] showed a big improvement of dosimetric results in $\mathrm{V}_{100}$ and $\mathrm{D}_{90}$ from $58.4 \%$ and $53.3 \%$ to $95.4 \%$ and $113 \%$, respectively, by comparing their pre-planning and intraoperative implant. The same comparison made by Wilkinson et al. [17] showed $\mathrm{V}_{100}$ increased from $76.2 \%$ to $84.9 \%$ and $\mathrm{D}_{90}$ increased from $82.8 \%$ to 94.1\%. Shanahan et al. [18] compared their pre-planning with the hybrid technique and the improvement of $\mathrm{V}_{100}$ and $\mathrm{D}_{90}$ were from $86 \%$ and $91 \%$ to $91 \%$ and $103 \%$, respectively. The implant transition in this study shows that the proposed technique is an effective intra-operative implantation method as well.

Although this initial study seems promising, the post dosimetric results of our $V_{100}$ and $D_{90}$ are still low compared to other reports, e.g., the Seattle [11], Mt. Sinai [5] and MSKCC [8] studies, which are listed in Table 3. We attempted not to overplan the target to avoid $\mathrm{D}_{90}$ of $180 \mathrm{~Gy}$ $(125 \%)$ or $\mathrm{V}_{150}$ of $60 \%$ as these are factors found to be related to urethral stricture [19-21]. Even $\mathrm{D}_{90}$ greater than $160 \mathrm{~Gy}(110 \%)$ was not allowed in our implantation since it correlates to rectal complication [22,23] and erectile dysfunction [24]. These considerations limit our current planning with conservative constraints and are set at $\mathrm{D}_{90} \sim 100 \%$ and $\mathrm{V}_{150}<45 \%$. Compared to the aforementioned reports, our current criteria restrict us to under-plan slightly. One way to improve the results of our implantation is to escalate our $\mathrm{D}_{90}$ and $\mathrm{V}_{100}$ in OR's pre-plan. With test plans of increasing the original activity by $5-10 \%$ such that the mean activity/(prostate volume) increased from $0.97 \mathrm{U} / \mathrm{cc}$ $(\mathrm{mCi} / \mathrm{cc})$ to $1.04 \mathrm{U} / \mathrm{cc}(0.82 \mathrm{mCi} / \mathrm{cc})$ in the post dosimetric results of $A r m B$, the $V_{100}, D_{90}$ and $V_{150}$ become $89.9 \pm$ $1.9,100.2 \pm 3.4$ and 57.4 \pm 4.6 , respectively. Based on these results and other institute's criteria, we may enhance our post dosimetric coverage by changing our planning constraints to $100 \%<\mathrm{D}_{90}<110 \%, \mathrm{~V}_{100}>95 \%$ and $\mathrm{V}_{150}<50 \%$.

Intraoperative optimization prevents the loss of geometric fidelity between pre- and in-OR prostate volumes, however, it cannot prevent the volume shape change during needle insert and seed loading. To keep higher $\mathrm{D}_{90}, \mathrm{~V}_{100}$, and low $\mathrm{V}_{150}$ values at the same time, dynamic calculation with updating of needle and seed position provide a better way to adapt the shape change during loading.

\section{Conclusions}

We have progressively moved our prostate seed implantation technique from a strictly pre-planned approached to an intraoperative optimization of the pre-plan. Our results demonstrate that the intraoperative modification provides superior target coverage while maintaining low rectal doses and a minimal increase in operative time ( $\sim 10 \mathrm{~min}$ utes). Without modifying the seed distribution in the OR, the planning $\mathrm{V}_{100}$ and $\mathrm{D}_{90}$ degraded by $6.4 \%$ and $9.3 \%$, respectively. With modification, the same numbers of seeds and needles could maintain a similar planning $V_{100}$ and $D_{90}$ between pre-plan and OR plan. The improvement of $\mathrm{V}_{100}$ and $\mathrm{D}_{90}$ in the post plan was $4.0 \%$ and $5.7 \%$, respectively, with less variation (reduced $40 \sim 50 \%$ standard deviation). In addition, to preserving the advantage of the cost-effective seed ordering and efficient OR implantation, our adaptive intraoperative optimization demonstrates increased accuracy and precision compared to the pre-planned technique.

\section{References}

1. Frank SJ, Arterbery VE, Hsu I et al. American College of Radiology Appropriateness Criteria permanent source brachytherapy for prostate cancer. Brachytherapy 2011; 10: 357-362.

2. Matzkin H, Kaver I, Bramante-Schreiber et al. Comparison between two idone-125 brachytherapy implant techniques: pre-planning and intra-operative by various dosimetry quality indicators. Raidiother Oncol 2003; 68: 289-294.

3. Gewanter RM, Wuu CS, Laguna JL et al. Intraoperative preplanning for transperineal ultrasounds-guided permanent prostate brachytherapy. Int J Radiat Oncol Biol Phys 2000; 48: 377-380.

4. Stock RG, Stone NN, Wesson MF et al. A modified technique allowing interactive ultrasound-guided three-dimensional transperineal prostate implantation. Int J Radiat Oncol Biol Phys 1995; 32: 219-225.

5. Stone NN, Hong S, Lo YC et al. Comparison of intraoperative dosimetric implant representation with postimplant dosimetry in patients receiving prostate brachytherapy. Brachytherapy 2003; 2: 17-25.

6. Zelefsky MJ, Yamada Y, Cohen G et al. Postimplantation dosimetric analysis of permanent transperineal prostate implantation: Improved dose distributions with an intraoperative computer-optimized conformal planning technique. Int J Radiat Oncol Biol Phys 2000; 48: 601-608.

7. Zelefsky MJ, Yamada Y, Cohen GN et al. Intra-operative realtime planned conformal prostate brachytherapy: Post-implantation dosimetric outcome and clinical implications. Radiother Oncol 2007; 84: 185-189.

8. Potters L, Calguaru E, Thoron KB et al. Toward a dynamic real-time intraoperative permanent prostate brachytherapy methodology. Brachytherapy 2003; 2: 172-180.

9. Meijer GJ, Berg HA, Hurkmans CW et al. Dosimetric comparison of interactive planned and dynamic dose calculated prostate seed brachytherapy. Radiother Oncol 2006; 80: 378-384.

10. Blasko JC, Radge H, Schumacher D. Transperineal percutaneous iodine-125 implantation for prostatic carcinoma using transrectal ultrasound and template guidance. Endocuriether Hyperthermia Oncol 1987; 3: 131-139.

11. Grimm P, Blasko J, Sylvester JE et al. Ten year biochemical (prostate-specific antigen) control of prostate cancer with 125-I brachytherapy. Int J Radiat Oncol Biol Phys 2001; 51: 31-40.

12. Sylvester JE, Grimm PD, Eulau SM et al. Permanent prostate brachytherapy preplanned technique: The modern Seattle method step-by-step and dosimetric outcomes. Brachytherapy 2009; 8: 197-206.

13. Lawton CA, DeSilvio M, Lee WR et al. Results of a phase II trial of transrectal ultrasound-guided permanent radioactive implantation of the prostate for definitive management of localized adenocarcinoma of the prostate (Radiation Therapy Oncology Group 98-05). Int J Radiat Oncol Biol Phys 2007; 67: 39-47.

14. Zelefsky MJ, Kuban DA, Levy LB et al. Multi-institutional analysis of long-term outcome for stage T1-T2 prostate cancer treated with permanent seed implantation. Int J Radiat Oncol Biol Phys 2007; 67: 327-333.

15. Nag S, Ciezki JP, Cormack R et al. Intraoperative planning and evaluation of permanent prostate brachytherapy: report 
of the American Brachytherapy Society. Int J Radiat Oncol Biol Phys 2001; 51: 1422-1430.

16. Ishiyama $\mathrm{H}, \mathrm{Nakamura} R$, Satoh $\mathrm{T}$ et al. Differences between intraoperative ultrasound-based dosimetry and postoperative computed tomography-based dosimetry for permanent interstitial prostate brachytherapy. Brachytherapy 2010; 9: 219-223.

17. Wilkinson DA, Lee EJ, Ciezki JP et al. Dosimetric comparison of pre-planned and OR-planned prostate seed brachytherapy. Int J Radiat Oncol Biol Phys 2000; 48: 1241-1244.

18. Shanahan TG, Nanavati PJ, Mueller PW et al. A comparison of permanent prostate brachytherapy techniques: preplan vs. hybrid interactive planning with posimplant analysis. Int J Radiat Oncol Biol Phys 2002; 53: 490-496.

19. Desai J, Stock RG, Stone NN et al. Acute urinary morbidity following I-125 interstitial implantation of the prostate gland. Radiat Oncol Invest 1998; 6: 135-141.

20. Stock RG, Stone NN, Dahlal M et al. What is the optimal dose for 125I prostate implants? A dose-response analysis of biochemical control, post treatment prostate biopsies, and longterm urinary symptoms. Brachytherapy 2002; 1: 83-89.

21. Merrick GS, Butler WM, Tollenaar BG et al. The dosimetry of prostate brachytherapy-induced urethral strictures. Int J Radiat Oncol Biol Phys 2002; 52: 461-468.

22. Snyder KM, Stock RG, Hong SM et al. Defining the risk of developing grade 2 proctitis following 125 I prostate brachytherapy using a rectal dose-volume histogram analysis. Int J Radiat Oncol Biol Phys 2001; 50: 335-341.

23. Han BH, Wallner KE. Dosimetric and radiographic correlates to prostate brachytherapy related rectal complications. Int I Cancer 2001; 96: 372-378.

24. Stock RG, KAO J, Stone NN. Penile erectile function after permanent radioactive seed implantation for treatment of prostate cancer. J Urology 2001; 165: 436-439. 